\title{
Caracterización de los ácidos grasos y actividad antimicrobiana del extracto en metanol de Holothuria princeps (Holothuriida: Holothuriidae)
}

\author{
Yeinner Quiroz Lobo*1, Gilmar Santafé Patiño ${ }^{1} \&$ Jorge A. Quirós-Rodríguez ${ }^{1}$ \\ 1. Grupo de Química de los Productos Naturales (PRONAT), Universidad de Córdoba, Cra. 6 N $^{\circ}$ 74-103 Montería, \\ Colombia; yeinerquirozlobo93@gmail.com,gsantafe@correo.unicordoba.edu.co,jquiros@correo.unicordoba.edu.co \\ * Correspondencia
}

Recibido 22-IV-2020. Corregido 19-VIII-2020. Aceptado 01-X-2020.

\begin{abstract}
Characterization of fatty acids and antimicrobial activity of the methanol extract of Holothuria princeps (Holothuriida: Holothuriidae). Introduction: In recent decades, studies related to the search and characterization of bioactive molecules in marine organisms have increased exponentially, demonstrating the enormous wealth of secondary metabolites of diverse structural composition that cannot be found in organisms present in the terrestrial environment. A significant number of the new marine natural compounds discovered have contributed to solving some of the problems of humanity, mainly those related to human health. Objective: The purpose of this research is to evaluate the bactericidal and fungicidal activities of the methanolic extract of sea cucumber Holothuria princeps collect from the bay of Cispata in the Colombian Caribbean, in addition to chemically identifying its fatty acids. Methods: A methanolic extraction was performed from the collected biological material, by the cold maceration method. The extract obtained was fractionated using chromatographic techniques and the fatty acids were obtained, which were derivatized and identified by means of gas chromatography in coupling with mass spectrometry. The antibacterial and antifungal activities of the methanolic extract of Holothuria princeps was performed through the microdilution method against reference strains and clinical isolates. Results: We found 16 fatty acids present in Holothuria princeps according to the analysis of their mass spectra. Antibacterial activity showed that Enterococcus faecalis was the most susceptible to the extract at low concentrations, while Pseudomonas aeruginosa was the highest at the higher concentrations. In antifungal treatment, the fungus with the highest inhibition was the clinical isolate of Candida albicans (blood sample). Conclusions: Taking into account previous studies in the genus Holothuria, it is considered that the environment plays a fundamental role in the presence and diversity of fatty acids. The evaluation of the antibacterial activity against reference strains of Escherichia coli, Pseudomonas aeruginosa, Staphylococcus aureus, Klebsiella pneumoniae and Enterococcus faecalis demonstrated the existence of a considerable effect in the reduction of bacterial growth by the extracts applied, mainly at low concentrations (less than $1000 \mathrm{ppm}$ ). On the other hand, the antifungal activity against the reference strain of Candida albicans and the clinical isolates of Candida albicans (blood sample) and Candida krusei (catheter sample), the extract showed that the best results were presented at higher concentrations (above $1500 \mathrm{ppm}$ ).
\end{abstract}

Key words: antibacterial; antifungic; Echinodermata; lipids; chromatography; mass Spectrometry.

Quiroz Lobo, Y., Santafé Patiño, G., \& Quirós-Rodríguez, J.A. (2021). Caracterización de los ácidos grasos y actividad antimicrobiana del extracto en metanol de Holothuria princeps (Holothuriida: Holothuriidae). Revista de Biología Tropical, 69(1), 36-44. DOI 10.15517/rbt.v69i1.41518

Durante millones de años, la vida marina ha producido una inmensa variedad de compuestos químicos de exclusivas estructuras y sofisticación (Fernández, Balmaseda,
\& Regueira, 2015). Desde esta perspectiva y teniendo en cuenta que los mares cubren la mayor parte del planeta, la bioprospección marina en comparación con la terrestre, puede 
considerarse como la forma más efectiva y prometedora en cuanto al posible descubrimiento de nuevos compuestos bioactivos (Rizzo \& Lo Giudice, 2018). Los invertebrados marinos registran una gran diversidad de metabolitos secundarios, alrededor de 10000 nuevos compuestos químicos son nombrados, estudiados y descritos anualmente (Valdés, Pérez, \& Colom, 2010; Birks, 2015; Carroll, Copp, Davis, Keyzers, \& Prinsep, 2019). En las últimas décadas se evidenció un continuo desarrollo de fármacos derivados de sustancias aisladas de organismos marinos (Sima \& Vetvicka, 2011; Piñón, García, Mendiola, Pérez, \& Monzote, 2015). Un gran número de invertebrados viven en íntima asociación con otros animales o plantas o microorganismos, factor que contribuye al incremento de una gran variedad de metabolitos secundarios biológicamente activos (Ramasami et al., 2016).

Como parte de los invertebrados, los holoturoideos, son organismos que habitan todos los ambientes marinos, algunas especies pueden desplegar capacidades natatorias (permanentes o facultativas) en el ambiente pelágico, mientras que otras ocupan distintos sustratos en hábitats bentónicos (Martins, 2012). Estos organismos, mezclan y consumen el material orgánico de los sedimentos, permitiendo así, la entrada del oxígeno, contribuyendo a la estructuración del hábitat y el reciclaje de la materia orgánica (Schneider et al., 2011; Purcell, Conand, Uthicke, \& Byrne, 2016). A lo largo de la historia, la pesca de holoturoideos ha sido extensiva, debido principalmente a su amplio uso en la medicina tradicional asiática dadas las numerosas aplicaciones contra la hipertensión, el asma, el reumatismo y otras lesiones (Wang et al., 2012). Estos beneficios biomedicinales se atribuyen a la presencia de componentes químicos, como esteroles, triterpenos, sulfatos de condroitina y glicosaminoglicanos (Baharara, Amini, Kerachian, \& Soltani, 2014).

Otros compuestos de interés sintetizados por los pepinos de mar, son los péptidos y los ácidos grasos. Los primeros, son importantes compuestos naturales biológicamente activos que tienen un alto potencial de valores nutracéuticos y medicinales debido a su amplio espectro de bioactividades, como son las actividades antimicrobiana, antiviral, antitumoral, antioxidante, entre otras (Chi, Cheung, $\mathrm{Ng}, \&$ Wong, 2015). Por su parte, los ácidos grasos se encuentran entre los principales componentes de las membranas celulares y son fundamentales en el mantenimiento de señales nerviosas a lo largo del sistema neuronal (Giménez, 1998). Estos compuestos están involucrados en varias vías bioquímicas y son una fuente importante de energía, son componentes estructurales de las membranas celulares e influyen en la permeabilidad y tráfico de compuestos celulares, la actividad de las proteínas y las rutas de señalización bioquímica (Goncalves et al., 2016).

Antes del descubrimiento y la utilización de los antimicrobianos por parte de la sociedad, las enfermedades infecciosas eran la principal causa de muerte del ser humano, y continúan siéndolo en países en vías de desarrollo (Aties, Duret, Tabío, \& Pérez, 2017). Los microorganismos han desarrollado nuevas estrategias que les proveen resistencia a la acción de fármacos, por lo que existe una prioridad de salud pública para explorar y desarrollar agentes antimicrobianos naturales más efectivos, con menores efectos secundarios, buena biodisponibilidad y de toxicidad mínima (Pérez, Falqué, \& Dominguez, 2016). Por lo anterior, muchos organismos marinos constituyen una potencial fuente de antimicrobianos capaces de combatir infecciones (Cusimano et al., 2019), en ese sentido, los extractos de especies de pepinos de mar, han demostrado una importante bioactividad (Sellem, Brahmi, Mnasser, Rafrafi, \& Bouhaouala-Zahar, 2017).

A pesar de la gran cantidad de compuestos químicos aislados en las últimas décadas a partir de pepinos de mar (Costa Leal, Puga, Serôdio, Gomes, \& Calado, 2012; Santafé, Guzmán, \& Torres, 2014; Boufridi \& Quinn, 2018 ), en muchas especies como Holothuria (Theelothuria) princeps Selenka, 1867, son muy pocos los reportes acerca de su composición química y estudios de bioprospección. Es por eso, que en este trabajo se identificaron los ácidos grasos presentes en especímenes de 
$H$. princeps recolectados en la bahía de Cispatá, Colombia, mostrando los resultados encontrados al cuantificar la actividad antimicrobiana de su extracto metanólico.

\section{MATERIALES Y MÉTODOS}

Área de estudio: Los especímenes de Holothuria princeps fueron recolectados en el sector Banco de Arena, bahía de Cispatá en el Caribe Colombiano $\left(09^{\circ} 25^{\prime} 26.15^{\prime \prime} \mathrm{N}\right.$

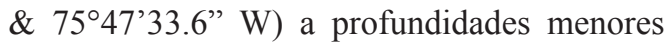
a $3 \mathrm{~m}$. El sector se caracteriza por presentar un sustrato arenoso y fango arenoso, lo cual brinda disponibilidad de sustrato importante para el establecimiento de gran variedad de invertebrados marinos, entre los que destacan equinodermos, moluscos, tunicados y esponjas (Nisperuza-Pérez, Quirós-Rodríguez, \& SolísMarín, 2019).

Los 10 ejemplares encontrados, fueron empacados individualmente en frascos de vidrio, con agua de mar y sal Epsom. Luego fueron almacenados en neveras de icopor y transportados al Laboratorio de Productos Naturales Marinos de la Universidad de Córdoba, donde se fotografiaron y observaron al estereoscopio, llevando a cabo un análisis detallado de su morfología.

Identificación del material biológico: La identificación taxonómica de la especie se realizó teniendo en cuenta la forma y combinación de los osículos calcáreos que constituyen el endoesqueleto de los pepinos de mar. Para esto, se realizó un corte de la piel usando una cuchilla de disección en la región ventral, dorsal y en los tentáculos, estos se ubicaron en portaobjetos separados, agregando varias gotas de hipoclorito de sodio hasta disolver la muestra de tejido (Ríos-Jara et al., 2013), posteriormente las muestras fueron observadas al microscopio ocular Leica Dm300 a un aumento de 10-40X. Uno de los ejemplares se determinó como material de referencia, el cual fue depositado en el Laboratorio de Zoología de la Universidad de Córdoba (LZUC-ECH 00112).
Preparación del material biológico: De los especímenes recolectados de $H$. princeps, 8 fueron cortados enteros en pequeños trozos ( $350 \mathrm{~g}$ peso vivo) y se sometieron a maceración en frío con Metanol en ausencia de luz por 72 horas, luego se filtró y concentró bajo el principio de destilación a presión reducida a $48^{\circ} \mathrm{C}$, obteniendo el extracto metanólico (2.5 g).

Fraccionamiento cromatográfico: El extracto metanólico (1.5 g) fue sometido a cromatografía de columna (CC) usando sistema de elución Bencina: Acetato de Etilo (7:1), las subfracciones obtenidas se monitorearon por cromatografía de capa delgada (CCD), usando patrones de ácido oleico, revelándose con luz ultravioleta y vapores de Yodo (Pastrana, Santafé, \& Quirós, 2016). Las subfracciones con $\mathrm{Rf}$ similar al ácido oleico se reunieron y se concentraron a presión reducida, logrando la fracción de ácidos grasos (C. Pérez et al., 2016).

Derivatización de ácidos grasos: $100 \mathrm{mg}$ de ácidos grasos se sometieron a reflujo con 5 $\mathrm{ml}$ de solución al $12 \%$ de Trifluoruro de Boro en Metanol $\left(\mathrm{BF}_{3} / \mathrm{MeOH}\right)$ por 3 horas a $80^{\circ} \mathrm{C}$. Luego se verificó la esterificación por $\mathrm{CCD}$ usando el mismo sistema anterior. La mezcla de reacción se extrajo con Diclorometano y se concentró a $38{ }^{\circ} \mathrm{C}$ (Guzmán, Quiroz, Angulo Ortiz, Torres, \& Santafé, 2014). Los ésteres metílicos obtenidos se purificaron por $\mathrm{CC}$ y posteriormente se analizaron por cromatografía de gases acoplado a espectrometría de masas (CG-EM) (Fuentes et al., 2013)

Identificación de los compuestos aislados: Los compuestos aislados fueron identificados a partir de los análisis de los espectros de masas, obtenidos por impacto electrónico, los cuales fueron estudiados, elucidados y comparados con los reportados en la bibliografía especializada (Pastrana Franco, Santafé Patiño, \& Sánchez Romero, 2019).

Ensayos de la actividad antimicrobiana: La actividad antimicrobiana de los extractos a concentraciones entre 25 y 2500 ppm (diluidos 
en Dimetilsulfóxido al $10 \%$ ), se evaluó frente a cepas de referencia de Escherichia coli (ATCC 25 922), Pseudomona aeruginosa (ATCC 27 852), Staphylococcus aureus (ATCC 29 213), Klebsiella pneumoniae (ATCC 10 031), Enterococcus faecalis (ATCC 29 212) y Candida albicans (ATCC 10 231); además de aislados clínicos de Candida albicans (Sangre) y Candida krusei (Catéter). Se empleó el método de microdilución, para lo cual se utilizaron microplacas ELISA de 96 pozos, sobre los que se adicionaron $50 \mu \mathrm{l}$ de los extractos y $50 \mu \mathrm{lde}$ la suspensión microbiana previamente estandarizada. Las microplacas fueron selladas con adhesivos e incubadas a $36{ }^{\circ} \mathrm{C}$ para bacterias y $27{ }^{\circ} \mathrm{C}$ para hongos, durante 24 horas. Tras la incubación, el crecimiento microbiano se evaluó midiendo los valores de absorbancia a $630 \mathrm{~nm}$ en el lector de ELISA. Como control positivo se utilizó Fluconazol a 500 ppm, para hongos y Ciprofloxacina a 500 ppm para bacterias (Méndez, Angulo, \& Contreras, 2016). Se calculó el porcentaje de reducción del crecimiento microbiano por parte del extracto y del control positivo $(\% \mathrm{R})$ utilizando la ecuación 1, para determinar el grado de inhibición de los extractos y del control positivo, donde $\mathrm{A}(\mathrm{CZ})$ es la absorbancia del control de crecimiento, $\mathrm{A}(\mathrm{M})$ es la absorbancia de la muestra y $\mathrm{A}(\mathrm{CP})$ la absorbancia del control positivo (Mohammadi \& Rohloff, 2016).

$$
\% \mathbf{R}=\frac{\mathrm{A}(\mathrm{CZ})-\mathrm{A}(\mathrm{M} \circ \mathrm{OCP})}{\mathrm{A}(\mathrm{CZ})} \times 100 \%
$$

Ecuación 1: \% de Reducción del crecimiento bacteriano.

Análisis estadístico: Para la determinación de la actividad antimicrobiana por el método de microdilución, se realizó una prueba de Shapiro-Wilk para la determinación de la normalidad y la homogeneidad de varianza. Además, se realizó un análisis de varianza no paramétrico de Kruskal Wallis haciendo partición por extractos y por concentración usando el software estadístico InfoStat versión 2018e.

\section{RESULTADOS}

Identificación de ácidos grasos: A partir de la cromatografía de gases, y por medio del análisis de los espectros de masas de impacto electrónico de los compuestos encontrados, se identificaron de 16 compuestos en la especie Holothuria princeps, los cuales son mostrados en la Tabla 1.

La composición de los ácidos grasos lineales $(75 \%)$ se encuentran en mayor proporción que los ramificados $(25 \%)$ en la subfracción lipídica. En cuanto a las insaturaciones, es evidente la prevalencia de ácidos grasos saturados (AGS) $(68.75 \%)$ sobre los monoinsaturados (AGMI) (31.25\%), no se encontraron compuestos poliinsaturados.

Actividad antibacteriana: Las concentraciones del extracto metanólico de $H$. princeps más efectivas frente a cada uno de los microorganismos tratados se muestran en la Tabla 2, el porcentaje de reducción del crecimiento microbiano a esas concentraciones y su comparación con el control positivo a $500 \mathrm{ppm}$ se presenta en la Fig. 1.

Todos los tratamientos bacterianos respondieron distinto ante diferentes concentraciones del extracto metanólico de ácidos grasos de Holothuria princeps y se observó que los resultados no siguieron un comportamiento normalizado (Shapiro-Wilk, valor-P: 0.04144). Se resaltan la inhibición del 67 y $56 \%$ del crecimiento de las cepas de $K$. pneumoniae y $E$. faecalis respectivamente, por parte del extracto a 25 ppm, así como la inhibición a 50 ppm frente a $E$. coli en donde el efecto es casi el doble del control positivo. En cuanto a los hongos, se observó que, en ninguna concentración del extracto, el porcentaje de reducción de la cepa de referencia de $C$. albicans (ATCC 10 231) supera al Fluconazol a 500 ppm; también se evidenció que a más altas concentraciones se presentan menores absorbancias. Además, se pudo establecer que, en la mayoría de las concentraciones utilizadas, el aislado clínico de $C$. albicans (sangre) fue el hongo más susceptible al efecto del extracto. 
TABLA 1

Ácidos grasos identificados

TABLE 1

Identified fatty acids

\begin{tabular}{|c|c|c|}
\hline No & Compuesto & $\mathrm{M}^{+}$ \\
\hline 1 & Ester metílico del ácido tetradecanoico & 242 \\
\hline 2 & Ester metílico del ácido 13-metiltetradecanoico & 256 \\
\hline 3 & Ester metílico del ácido pentadecanoico & 256 \\
\hline 4 & Ester metílico del ácido 9-Hexaddecenoico & 268 \\
\hline 5 & Ester metílico del ácido hexadecanoico & 270 \\
\hline 6 & Ester metílico del ácido 10-metilhexadecanoico & 284 \\
\hline 7 & Ester metílico del ácido 10-heptadecenoico & 282 \\
\hline 8 & Ester metílico del ácido heptadecanoico & 284 \\
\hline 9 & Ester metílico del ácido 11-octadecenoico & 296 \\
\hline 10 & Ester metílico del ácido octadecanoico & 298 \\
\hline 11 & Ester metílico del ácido 2-undecilciclopropanopentanoico & 310 \\
\hline 12 & Ester metílico del ácido nonadecanoico & 312 \\
\hline 13 & Ester metílico del ácido 11-eicosenoico & 324 \\
\hline 14 & Ester metílico del ácido eicosanoico & 326 \\
\hline 15 & Ester metílico del ácido 2-octilciclopropanodecanoico & 340 \\
\hline 16 & Ester metílico del ácido heneicosanoico & 340 \\
\hline
\end{tabular}

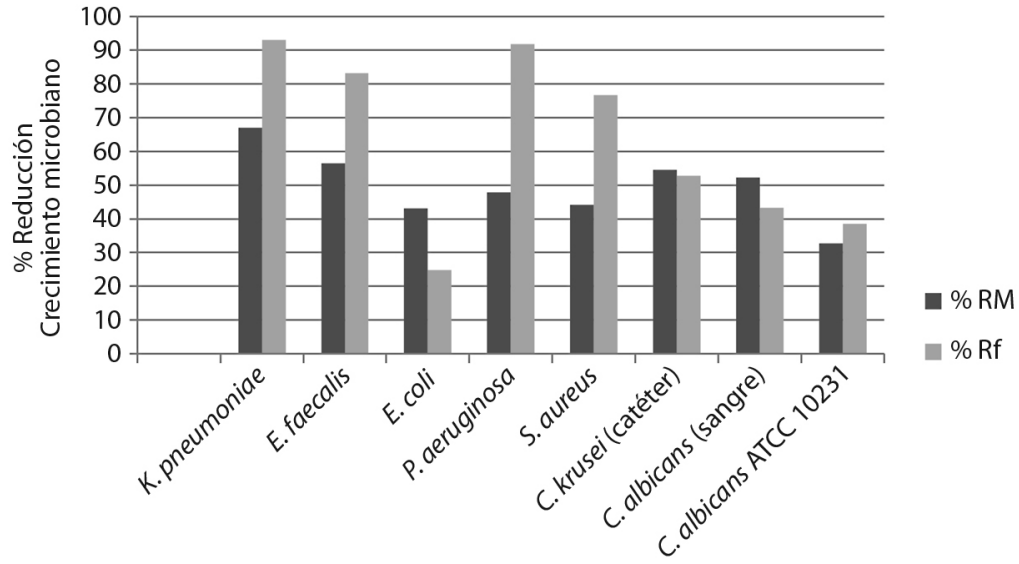

Microorganismos evaluados

Fig. 1. Porcentaje de reducción del extracto y del control positivo frente a cada una de los microorganismos tratados (\% RM: es el porcentaje de reducción del crecimiento microbiano de $H$. princeps; \% Rf: porcentaje de reducción del control positivo a $500 \mathrm{ppm}$ ).

Fig. 1. Percentage reduction of the extract and positive control against each of the microorganisms treated (\% RM: percentage of reduction of the microbial growth of $H$. princeps; \% Rf: percentage of reduction of the positive control at $500 \mathrm{ppm})$.

\section{DISCUSIÓN}

Los ácidos grasos en los pepinos de mar son componentes esenciales, responsables de la reparación de tejidos y las propiedades de regeneración (Ibrahim, Wong, Mohamed, \& Mohamed, 2018). La composición de los ácidos grasos en los pepinos de mar puede variar por 
TABLA 2

Concentración más efectiva del extracto en metanol de $H$. princeps frente a cada uno de los microorganismos utilizados

TABLE 2

Most effective concentration of methanolic extract of $H$. princeps against each of the microorganisms used

\begin{tabular}{lcccc}
\multicolumn{1}{r}{ Microrganismo } & Concentración $(\mathrm{ppm})$ & Absorbancia & Cf \\
K. pneumoniae & 25 & 0.44 & 1.34 & 0.09 \\
E. faecalis & 25 & 0.63 & 1.44 & 0.24 \\
E. coli & 50 & 0.54 & 0.94 & 0.71 \\
P. aeruginosa & 750 & 0.62 & 1.18 & 0.10 \\
S. aureus & 750 & 0.49 & 0.87 & 0.20 \\
C. krusei (catéter) & 1750 & 0.55 & 1.21 & 0.57 \\
C. albicans (sangre) & 2250 & 0.60 & 1.26 & 0.71 \\
C. albicans ATCC 10231 & 2250 & 0.70 & 1.04 & 0.64 \\
\hline
\end{tabular}

M: absorbancias de las muestras, C: absorbancias del control de crecimiento y Cf: absorbancias del control positivo.

diferentes factores, por la disponibilidad de los ingredientes de la dieta y las fuentes alimenticias (Al Azad, Shaleh, \& Siddiquee, 2017), por la temperatura ambiente del hábitat (Taboada, Gonzalez, \& Rodriguez, 2003), e incluso por la profundidad en la que viven, donde su síntesis está relacionada con el mantenimiento de la fluidez de la membrana a altas presiones y bajas temperaturas (Mehmet, Sevgili, Tufan, Emre, \& Ko, 2011). A diferencia de estudios anteriores realizados en especies del género Holothuria, en la subfracción lipídica estudiada de $H$. princeps se encontró la prevalencia de ácidos grasos saturados sobre los monoinsaturados y no se evidenciaron ácidos grasos poliinsaturados. Es de resaltar, que los componentes dominantes entre los ácidos grasos saturados y monoinsaturados de $H$. princeps, fueron el ácido hexadecanoico (palmítico) y el ácido 11-eicosenoico respectivamente, lo que coincide con otras especies de pepinos de mar (Bordbar, Anwar, \& Saari, 2011).

Investigaciones recientes, demuestran que productos naturales extraídos de pepinos de mar como saponinas, triterpenos y ácidos grasos, han sido probados en varios estudios como posibles agentes antimicrobianos (Nazemi et al., 2020). En este trabajo se evidenció una disminución del crecimiento bacteriano a concentraciones bajas del extracto, siendo el efecto en algunos casos, mayor que el mostrado por la Ciprofloxacina a $500 \mathrm{ppm}$, resultados que coinciden con extractos orgánicos de otras especies del género, como $H$. parva (Ebrahimi et al., 2018), H. scabra, H. leucospilota y $H$. atra (Abdallah \& Ibrahim, 2013). Sin embargo, en el extracto metanólico de H. princeps, Enterococcus faecalis y Pseudomonas aeruginosa fueron las bacterias más susceptibles, lo que contrasta con los resultados obtenidos por (Mashjoor \& Yousefzadi, 2016), donde la inhibición de estas bacterias fue mínima.

En estudios previos llevados a cabo con otras especies de Holothurios (Ismail et al., 2008; Santafé, Guzmán, \& Torres, 2014; Santos et al., 2016), se ha evidenciado que los extractos orgánicos poseen actividad fungicida contra ciertas cepas de hongos. En este sentido, el extracto metanólico de $H$. princeps logró reducir el crecimiento de los hongos C. albicans (ATCC 10 231), C. albicans (muestra de sangre) y C. krusei (muestra de catéter), principalmente a las concentraciones más altas (mayores a $1500 \mathrm{ppm}$ ), lo que sugiere la presencia de compuestos antifúngicos dentro del extracto.

Declaración de ética: los autores declaran que todos están de acuerdo con esta publicación y que han hecho aportes que justifican su autoría; que no hay conflic-to de interés de 
ningún tipo; y que han cumplido con todos los requisitos y pro-cedimientos éticos y legales pertinentes. Todas las fuentes de financiamiento se detallan plena y claramente en la sección de agradecimientos. El respectivo do-cumento legal firmado se encuentra en los archivos de la revista.

\section{RESUMEN}

Introducción: En las últimas décadas los estudios relacionados con la búsqueda y caracterización de moléculas bioactivas en organismos marinos han aumentado de una manera exponencial, lo que demuestra la enorme riqueza de metabolitos secundarios de diversa composición estructural que no pueden ser encontrados en organismos presentes en el medio terrestre. Estas nuevas moléculas halladas poseen numerosas actividades biológicas que ayudan a resolver muchos problemas que ha tenido el hombre a lo largo de su existencia, lo que las convierte en productos de gran importancia para la humanidad. Objetivo: El propósito de este estudio es la identificación de los ácidos grasos presentes en el pepino de mar Holothuria princeps recolectado en costas del Caribe colombiano, además del análisis de las actividades antibacterianas y antifúngicas de su extracto metanólico frente a cepas de referencia y aislados clínicos. Métodos: Del material biológico recolectado se realizó una extracción metanólica usando el método de maceración en frío. El extracto obtenido se fraccionó usando cromatografía en columna y se lograron obtener los ácidos grasos, los cuales fueron derivatizados e identificados por medio de cromatografía de gases en acople con espectrometría de masas. La actividad antibacteriana y antifúngica del extracto metanólico de Holothuria princeps se realizó a través del método de microdilución. Resultados: Los resultados arrojaron la identificación de 16 ácidos grasos presentes en Holothuria princeps de acuerdo con el análisis de sus espectros de masas. La actividad antibacteriana mostró que Enterococcus faecalis fue la bacteria más susceptible al efecto del extracto a bajas concentraciones, mientras que a las más altas lo fue Pseudomonas aerugino$s a$. A nivel general en el tratamiento antifúngico, el hongo que presentó una mayor inhibición fue el aislado clínico de Candida albicans (muestra de sangre). Conclusiones: Teniendo en cuenta estudios previos en organismos del mismo género, se puede considerar en cuanto a los ácidos grasos identificados, que el entorno juega un papel fundamental en la presencia y composición de estos compuestos. La evaluación de la actividad antibacteriana contra cepas de referencia de Escherichia coli, Pseudomonas aeruginosa, Staphylococcus aureus, Klebsiella pneumoniae y Enterococcus faecalis, demostró la existencia de un efecto considerable en la reducción del crecimiento bacteriano por parte de los extractos utilizados, principalmente a bajas concentraciones (menos de $1000 \mathrm{ppm}$ ). En cuanto a la actividad antifúngica frente a la cepa de referencia de Candida albicans y a los aislados clínicos de Candida albicans (muestra de sangre) y Candida krusei (muestra de catéter), el extracto mostró que los mejores resultados se presentaron concentraciones por encima de $1500 \mathrm{ppm}$.

Palabras clave: antibacteriano; antifúngico; equinodermos; lípidos; cromatografía; espectrometría de masas.

\section{REFERENCIAS}

Abdallah, H., \& Ibrahim, H. (2013). Antibacterial carotenoids of three Holothuria species in Hurghada, Egypt. The Egyptian Journal of Aquatic Research, 38(3), 185-194.

Al Azad, S., Shaleh, M., \& Siddiquee, S. (2017). Comparison of Fatty Acid and Proximate Composition between Holothuria edulis and Holothuria scabra Collected from Coastal Water of Sabah, Malaysia. Advances in Bioscence and Biotechnology, 8, 91-103.

Aties, L., Duret, Y., Tabío, M., \& Pérez, S. (2017). Los enzibióticos como alternativa terapéutica contra las enfermedades bacterianas The enzybiotics as therapeutic alternative against bacterial diseases. Medisan, 21(10), 3077-3083.

Baharara, J., Amini, E., Kerachian, M.A., \& Soltani, M. (2014). The osteogenic differentiation stimulating activity of Sea cucumber methanolic crude extraction on rat bone marrow mesenchymal stem cells. Iranian Journal of Basic Medical Sciences, 17(8), 626-631.

Birks, H. (2015). Biodiversity, Livelihood and Climate Change in the Himalayas. In P. Jha, K. Shrestha, R. Chaudhary, \& B. Shrestha (Eds.), Proceedings of International Conference on Biodiversity, Livelihood and Climate Change in the Himalayas (pp. 1-39). Kirtipur, Nepal: Mass Printers.

Bordbar, S., Anwar, F., \& Saari, N. (2011). High-Value Components and Bioactives from Sea Cucumbers for Functional Foods- A Review. Marine Drugs, 9, 1761-1805.

Boufridi, A., \& Quinn, R.J. (2018). Harnessing the Properties of Natural Products. Revisión Anual de Farmacología y Toxicología, 58, 451-470.

Chi, R., Cheung, F., Ng, T.B., \& Wong, J.H. (2015). Marine Peptides: Bioactivities and Applications. Marine Biotechnology, 13(7), 4006-4043.

Costa Leal, M., Puga, J., Serôdio, J., Gomes, N., \& Calado, R. (2012). Trends in the discovery of new marine natural products from invertebrates over the last two decades - where and what are we bioprospecting? PLOS ONE, 7(1), 1-15.

Cusimano, M.G., Spinello, A., Barone, G., Schillaci, D., Cascioferro, S., Magistrato, A., ... Vitale, M. (2019). 
A Synthetic Derivative of Antimicrobial Peptide Holothuroidin 2 from Mediterranean Sea Cucumber (Holothuria tubulosa) in the Control of Listeria monocytogenes. Marine Drugs, 17(3), 1-11.

Ebrahimi, H., Vazirizadeh, A., Nabipour, I., Najafi, A., Tajbakhsh, S., \& Nafisi Bahabadi, M. (2018). In vitro study of antibacterial activities of ethanol, methanol and acetone extracts from sea cucumber Holothuria parva. Iranian Journal of Fisheries Sciences, 17(3), 542-551.

Fernández, M., Balmaseda, I., \& Regueira, S. (2015). Bioactivos marinos en el tratamiento del cáncer. Revista Electronica Dr. Zolio E. Marinello Vidaurreta, 40(7), 1-9.

Fuentes, E., Rodríguez-Pérez, W., Guzmán, L., Alarcón, M., Navarrete, S., Forero-doria, O., \& Palomo, I. (2013). Mauritia flexuosa Presents In Vitro and In Vivo Antiplatelet and Antithrombotic Activities. Evidence-Based Complementary and Alternative Medicine, 2013, 1-11.

Giménez, C. (1998). Composición y estructura de la membrana neuronal : bases moleculares de su fisiología y su patología. Revista de Neurología, 26(150), 232-239.

Goncalves, A.M.M., Mesquita, A.F., Verdelhos, T., Coutinho, J.A.P., Marques, J.C., \& Goncalves, F. (2016). Fatty acids' profiles as indicators of stress induced by of a common herbicide on two marine bivalves species: Cerastoderma edule (Linnaeus , 1758) and Scrobicularia plana (da Costa , 1778). Ecological Indicators, 63(1), 657-672.

Guzmán, M., Quiroz, J., Angulo Ortiz, A., Torres, O., \& Santafé, G.G. (2014). Bioprospección e identificación de los ácidos grasos del pepino de mar Holothuria floridana. Evodia, 1(1), 39-46.

Ibrahim, N.I., Wong, S.K., Mohamed, I.N., \& Mohamed, N. (2018). Wound Healing Properties of Selected Natural Products. International Journal of Environmental Research and Public Health, 15(11), 2360.

Ismail, H., Lemriss, S., Ben Aoun, Z., Mhadhebi, L., Dellai, A., Kacem, Y., ... Bouraoui, A. (2008). Antifungal activity of aqueous and methanolic extracts from the Mediterranean sea cucumber, Holothuria polii. Journal de Mycologie Medicale, 18(1), 23-26.

Martins, L. (2012). Estudo Taxonômico dos Holothuroidea (Echinodermata) de águas rasas da costa Brasileira. Salvador, Brasil: Universidade Federal da Bahia.

Mashjoor, S., \& Yousefzadi, M. (2016). Holothurians antifungal and antibacterial activity to human pathogens in the Persian Gulf. Journal de Mycologie Medicale, 27(1), 46-56.

Mehmet, A., Sevgili, H., Tufan, B., Emre, Y., \& Ko, S. (2011). Proximate composition and fatty acid profile of three different fresh and dried commercial sea cucumbers from Turkey. International Journal of Food Science \& Technology, 46, 500-508.

Méndez, N., Angulo, A., \& Contreras, O. (2016). Actividad antibacteriana in vitro de Curcuma longa (Zingiberaceae) frente a bacterias nosocomiales en Montería, Colombia. Revista de Biología Tropical, 64(3), 1201-1208.

Mohammadi, M., \& Rohloff, J. (2016). Antibiofilm activity of essential oils and plant extracts against Staphylococcus aureus and Escherichia coli biofilms. Food Control, 61, 156-164.

Nazemi, M., Moradi, Y., Mortazavi, M.S., Mohebbi Nozar, S.L., Karimzadeh, R., \& Ghaffari, H. (2020). Isolation and identification of antibacterial compound from common name Holothuria leucospilota in the Persian Gulf. Iranian Journal of Fisheries Sciences, 19(5), 2493-2500.

Nisperuza-Pérez, C., Quirós-Rodríguez, J., \& Solís-Marín, F.A. (2019). Primeros registros e intervalos de distribución de Holothuria (Theelothuria) princeps Selenka , 1867 (Echinodermata : Holothuroidea : Holothuriidae ) en el golfo de Morrosquillo , Caribe Colombiano. Revista de Biología Marina y Oceanografia, 54(1), 139-143.

Pastrana Franco, O., Santafé Patiño, G., \& Sánchez Romero, E. (2019). Perfil lipídico y ensayos de las actividades antioxidante, insecticida y antialimentaria de la esponja marina Iotrochota birotulata (Iotrochotidae: Demospongiae ). Revista de Biología Tropical, 67, 213-223.

Pastrana, O., Santafé, G., \& Quirós, J. (2016). Actividad antioxidante del erizo de mar Mellita quinquiesperforata (Leske) e identificación de sus compuestos lipídicos mayoritarios. Actualidades Biológicas, 38(104), 15-22.

Pérez, C., Santafé, G., Torres, M., Torres, O., Montaño, M., \& Robledo, S. (2016). Composición lipídica y evaluación de las actividades antioxidante y leishmanicida del basidiomiceto Ganoderma sp . leishmanicidal activities of the basidiomycete Ganoderma sp. Revista Cubana de Plantas Medicinales, 21(3), 318-331.

Pérez, M.J., Falqué, E., \& Dominguez, H. (2016). Antimicrobial Action of Compounds from Marine Seaweed. Marine Drugs, 14(3), 1-38.

Piñón, A., García, M., Mendiola, J., Pérez, A., \& Monzote, L. (2015). Evaluación in vitro de extractos de invertebrados marinos frente a Leishmania amazonensis. Revista Cubana de Medicina Tropical, 67(3), 1-8.

Purcell, S.W., Conand, C., Uthicke, S., \& Byrne, M. (2016). Ecological roles of exploited sea cucumbers. Oceanografía y Biología Marina: Una Revisión Anual, 54, 367-386. 
Ramasami, P., Gupta, M., Sabina, B., Laulloo, J., Li, H., \& Wah, K. (2016). Crystallizing Ideas - The Role of Chemistry. New York, USA: Springer.

Ríos-Jara, E., Galván-Villa, C.M., Rodríguez-Zaragoza, F.A., López-Uriarte, E., Bastida-Izaguirre, D., \& Solís-Marin, F. (2013). Los equinodermos (Echinodermata) de bahía Chamela, Jalisco, México. Revista Mexicana de Biodiversidad, 84(1), 263-279.

Rizzo, C., \& Lo Giudice, A. (2018). Marine Invertebrates : Underexplored Sources of Bacteria Producing Biologically Active Molecules. Diversity, 52(10), 1-36.

Santafé, G., Guzmán, M., \& Torres, O. (2014). Triterpenos holostánicos con actividad antifúngica obtenidos del pepino de mar Holothuria floridana, recolectado en la bahía de cispatá, Córdoba-Colombia. Información Tecnológica, 25(2), 87-92.

Santos, R., Dias, S., Pinteus, S., Silva, J., Alves, C., Tecelao, C., ... Pombo, A. (2016). Sea cucumber Holothuria forskali, a new resource for aquaculture? Reproductive biology and nutraceutical approach. Aquaculture Research, 47(7), 2307-2323.

Schneider, K., Silverman, J., Woolsey, E., Eriksson, H., Byrne, M., \& Caldeira, K. (2011). Potential influence of sea cucumbers on coral reef $\mathrm{CaCO} 3$ budget: A case study at One Tree Reef. Journal of Geophysical Research: Biogeosciences, 116(4), 2-7.

Sellem, F., Brahmi, Z., Mnasser, H., Rafrafi, S., \& Bouhaouala-Zahar, B. (2017). Antimicrobial activities of coelomic fluid and body wall extracts of the edible Mediterranean sea cucumber Holothuria tubulosa Gmelin, 1790. Cahiers de Biologie Marine, 58(2), 181-188.

Sima, P., \& Vetvicka, V. (2011). Bioactive substances with anti-neoplastic efficacy from marine invertebrates: Porifera and Coelenterata. World Journal of Clinical Oncology, 2(11), 355-361.

Taboada, M.C., Gonzalez, M., \& Rodriguez, E. (2003). Value and effects on digestive enzymes and serum lipids of the marine invertebrate Holothuria forskali. Nutrition Research, 23, 1661-1670.

Valdés, O., Pérez, R., \& Colom, Y. (2010). Actividad antitumoral de los organismos marinos. Revista Electrónica de la Agencia de Medio Ambiente, 19(1), 1683-8904.

Wang, Y., Su, W., Zhang, C., Xue, C., Chang, Y., Wu, X., ... Wang, J. (2012). Protective effect of sea cucumber (Acaudina molpadioides) fucoidan against ethanol-induced gastric damage. Food Chemistry, 133(4), 1414-1419. 